Proceedings 
Ein stetig steigender Fundus an Informationen ist heute notwendig, um die immer komplexer werdende Technik heutiger Kraftfahrzeuge zu verstehen. Funktionen, Arbeitsweise, Komponenten und Systeme entwickeln sich rasant. In immer schnelleren Zyklen verbreitet sich aktuelles Wissen gerade aus Konferenzen, Tagungen und Symposien in die Fachwelt. Den raschen Zugriff auf diese Informationen bietet diese Reihe Proceedings, die sich zur Aufgabe gestellt hat, das zum Verständnis topaktueller Technik rund um das Automobil erforderliche spezielle Wissen in der Systematik der Konferenzen und Tagungen zusammen zu stellen und als Buch in Springer.com wie auch elektronisch in SpringerLink und Springer für Professionals bereit zu stellen.

Die Reihe wendet sich an Fahrzeug- und Motoreningenieure sowie Studierende, die aktuelles Fachwissen im Zusammenhang mit Fragestellungen ihres Arbeitsfeldes suchen. Professoren und Dozenten an Universitäten und Hochschulen mit Schwerpunkt Kraftfahrzeug- und Motorentechnik finden hier die Zusammenstellung von Veranstaltungen, die sie selber nicht besuchen konnten. Gutachtern, Forschern und Entwicklungsingenieuren in der Automobilund Zulieferindustrie sowie Dienstleistern können die Proceedings wertvolle Antworten auf topaktuelle Fragen geben. 
Gerhard Tecklenburg

Herausgeber

\section{Karosseriebautage Hamburg 2016}

14. ATZ-Fachtagung

Springer Vieweg 
Herausgeber

Prof. Dr. Gerhard Tecklenburg

HAW Hamburg

Fakultät Technik und Informatik

Hamburg, Deutschland

ISSN 2198-7432

ISSN 2198-7440 (electronic)

Proceedings

ISBN 978-3-658-14143-1

ISBN 978-3-658-14144-8 (eBook)

DOI 10.1007/978-3-658-14144-8

Die Deutsche Nationalbibliothek verzeichnet diese Publikation in der Deutschen Nationalbibliografie; detaillierte bibliografische Daten sind im Internet über http://dnb.d-nb.de abrufbar.

Springer Vieweg

(C) Springer Fachmedien Wiesbaden 2016

Das Werk einschließlich aller seiner Teile ist urheberrechtlich geschützt. Jede Verwertung, die nicht ausdrücklich vom Urheberrechtsgesetz zugelassen ist, bedarf der vorherigen $\mathrm{Zu}-$ stimmung des Verlags. Das gilt insbesondere für Vervielfältigungen, Bearbeitungen, Übersetzungen, Mikroverfilmungen und die Einspeicherung und Verarbeitung in elektronischen Systemen.

Die Wiedergabe von Gebrauchsnamen, Handelsnamen, Warenbezeichnungen usw. in diesem Werk berechtigt auch ohne besondere Kennzeichnung nicht zu der Annahme, dass solche Namen im Sinne der Warenzeichen- und Markenschutz-Gesetzgebung als frei zu betrachten wären und daher von jedermann benutzt werden dürften.

Der Verlag, die Autoren und die Herausgeber gehen davon aus, dass die Angaben und Informationen in diesem Werk zum Zeitpunkt der Veröffentlichung vollständig und korrekt sind. Weder der Verlag noch die Autoren oder die Herausgeber übernehmen, ausdrücklich oder implizit, Gewähr für den Inhalt des Werkes, etwaige Fehler oder Äußerungen.

Gedruckt auf säurefreiem und chlorfrei gebleichtem Papier.

Springer Vieweg ist Teil von Springer Nature

Die eingetragene Gesellschaft ist Springer Fachmedien Wiesbaden GmbH 


\section{HERZLICH WILLKOMMEN}

Mobilität, Sicherheit, Komfort und Umwelt sind die technischen Hauptziele der Produktentwicklung im Automobilbau. Die Reduzierung des Kraftstoffverbrauchs und die Senkung der $\mathrm{CO}_{2}$-Emission stellen dabei Entwicklungs- und Fertigungsingenieure vor immer neue Herausforderungen.

Die Fahrzeugkarosserie - Exterieur und Interieur - hat mit ihrem Gewichtsanteil von ca. $55 \%$ wesentlichen Einfluss auf das Gesamtgewicht des Standardfahrzeugs. Die komplexe Karosserie bietet durch ihre Formen, ihre Funktionen der integrierten Systeme und ihr Zusammenwirken mit Antrieb, Fahrwerk, Insassen und Verkehrsraum vielfältige Ansätze für Fachdiskussionen und Lösungsansätze für die gestellten Anforderungen.

Leichtbau, innovative Werkstoffe und alternative Fertigungsverfahren stehen deshalb im Fokus der diesjährigen 14. Karosseriebautage im Terminal Tango am Hamburger Flughafen, wo sich die Karosseriebauexperten zum Erfahrungsaustausch treffen, um neueste Entwicklungen und Trends zu diskutieren.

Ziel dieser Tagung ist, die an der Entwicklung und Fertigung der Karosserie Beteiligten zu einem Erfahrungsaustausch zusammenzuführen und dazu beizutragen, dass die gestellten Aufgaben gemeinsam besser bewältigt werden können.

Im Namen des Wissenschaftlichen Beirats heißen wir Sie herzlich in Hamburg willkommen. Informieren Sie sich über die aktuellsten Themen und erweitern Sie Ihr Netzwerk!

Für den Wissenschaftlichen Beirat

Prof. Dr. Gerhard Tecklenburg

Wolfgang Siebenpfeiffer

HAW Hamburg

Leiter Wissenschaftlicher Beirat

Herausgeber ATZ | MTZ |

ATZelektronik | lightweight design 


\section{Statt halbes...}
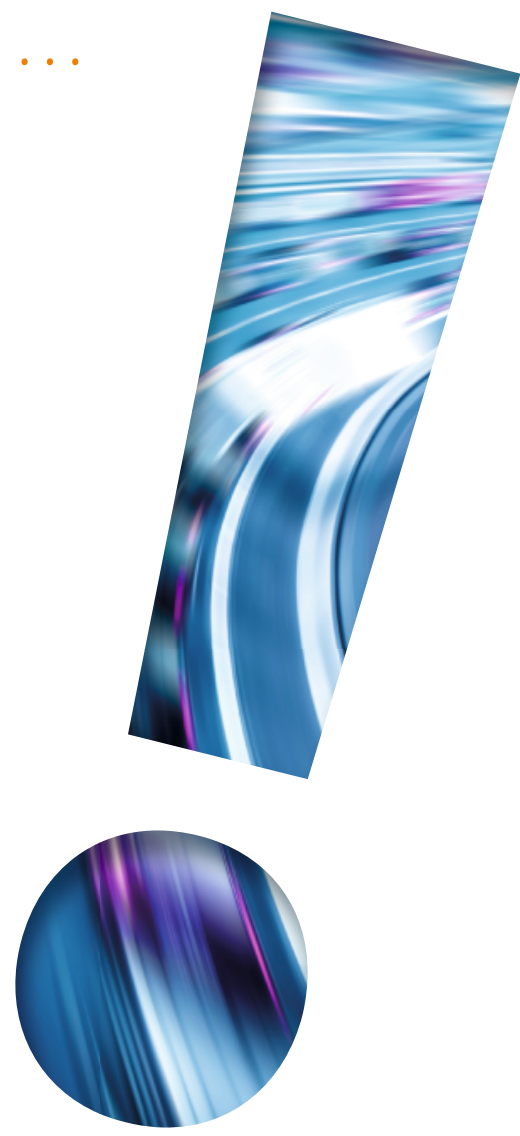

... bestes Wissen aus einer Hand!

Vom Internet der Dinge bis hin zu Compliance: Für Lösungen, die auf ganzer Länge überzeugen, braucht man umfassendes Wissen. Und das bietet Ihnen Springer Professional, die größte digitale Fachbibliothek für Wirtschaft und Technik. Mit über 1,2 Millionen Volltext-Dokumenten aus Fachzeitschriften und Fachbüchern angesehener Autoren und renommierter Verlage. Mit aktuellen Beiträgen und ständig neuen Inhalten. Mit intelligenten Suchfunktionen, unbegrenztem, mobilen Zugriff und vielen weiteren Vorteilen. Jetzt mehr erfahren und kostenlosen Testzugang sichern: www.springerprofessional.de

\section{Springer Professional}




\section{INHALT}

\section{FORSCHUNG UND ENTWICKLUNG}

Keynote-Vortrag |

Der Natur in die Karten geschaut -

Optimierungsverfahren aus dem Bereich der Bionik

Prof. Dr. Lothar Harzheim, Adam Opel AG

Funktionsintegrierte und bionisch optimierte

Fahrzeugleichtbaustruktur in flexibler Fertigung

Dr. Martin Hillebrecht, EDAG Engineering $\mathrm{GmbH}$;

Prof. Dr. Claus Emmelmann, LZN Laser Zentrum Nord GmbH

\section{KOMPONENTEN}

Scheinwerfer 2025 -

bionisch inspiriert und generativ gefertigt

Klaus Zander, D. Sokolov, W. Schwarz, M. Frohnapfel, EDAG Engineering $\mathrm{GmbH}$

Die Schiebetür - alles andere als konventionell

Andreas Cousin, Dr. J. Klingbeil, Ford-Werke GmbH

Entwicklung des Fahrzeugunterbodens vor dem Hintergrund $\mathbf{C O}_{2}$-Effizienz

Oliver Mende, Volkswagen AG 


\section{KAROSSERIEENTWICKLUNG}

Keynote-Vortrag |

Elektrisch angetriebene Stadtbusse -

Ausgangslage, Entwicklungsstand, Ausblick

Ulrich Sieg, ehem. Hamburger Hochbahn AG

Effiziente rechnerunterstützte Entwicklung von Karosseriekonzepten

Prof. Dr. Klemens Rother,

Fakultät Maschinenbau/Fahrzeugtechnik/Flugzeugtechnik, Hochschule München; Dr. W. Pohl, FCMS GmbH;

Dr. D. Schneider, Dynardo GmbH; U. Jankowski, Tecosim GmbH

MAN-Omnibusse - Rechnungs-Messungs-Vergleich

des dynamischen Umsturzes eines Gesamtfahrzeugs

Dr. Roland Krivachy, Dr. S. Guserle, L. Ginsberg,

MAN Truck \& Bus AG

\section{FAHRZEUG I}

Carbon Core - die neue BMW 7er Karosserie

Michael Ahlers, BMW Group

The All New Opel Astra Sports Tourer

Frank Saterdag, Gerald Creter, Adam Opel AG

Mercedes-Benz F015 - autonome Mobilität der Zukunft

Sylvain Wehnert, Daimler AG

Impulsvortrag | Auto und Karosserie vom hölzernen Kutschkasten zur selbsttragenden Verbundkarosserie

Erik Eckermann, AutoHistorica 


\section{KONZEPTE}

Passive Sicherheit instand gesetzter Pkw

Helge Kiebach, KTI GmbH \& Co. KG

Die Revolution in der automobilen Softwareentwicklung und ihre Auswirkungen auf das Interieur Heiko Herchet, trive.me - eine Marke der EDAG Engineering $\mathrm{GmbH}$

\section{ENTWICKLUNGSMETHODEN I}

Keynote-Vortrag | Virtuelles Testen von kohlefaserverstärkten Kunststoffbauteilen bei Airbus Dr. Wolfgang Wohlers, Airbus Operations $\mathrm{GmbH}$

Methoden zur Fahrersicht-Optimierung am Beispiel des neuen Opel ASTRA

Dr. Lars Woyna, Adam Opel AG

Batterieträger in Faserkunststoffverbundbauweise Nino Andricevic, Dr. Ing. h.c. F. Porsche AG;

A. Liebsch, Prof. Dr. M. Gude,

Institut für Leichtbau und Kunststofftechnik (ILK), TU Dresden

\section{ENTWICKLUNGSMETHODEN II}

BFast - systematische Ableitung von Ersatzlastfällen aus Betriebslasten Sascha Wörner, Prof. Dr. U. Jung, Kompetenzzentrum für Verkehr - Mobilität - Automotive, Technische Hochschule Mittelhessen

Die Konzeptentwicklung als Bindeglied der technischen Innovationen zum realen Design Andreas Orf, M. Duscha, AUDI AG 


\section{FAHRZEUG II}

Ford Focus RS - Rebirth of an Icon

Rolf Züchner, Ford-Werke $\mathrm{GmbH}$;

Tobias Fritze, EDAG Engineering $\mathrm{GmbH}$

Highlights des Porsche Boxster Spyder

Detlev Ranft, Kai Geißenhöner, Dr. Ing. h.c. F. Porsche AG

2016 Cadillac CT6 - Product Engineering

Robert N. Saje, General Motors Company, USA 


\section{REFERENTEN}

Michael Ahlers

BMW Group

Nino Andricevic

Dr. Ing. h.c. F. Porsche AG

Andreas Cousin

Ford-Werke $\mathrm{GmbH}$

Gerald Creter

Adam Opel AG

Erik Eckermann

AutoHistorica

Prof. Dr. Claus Emmelmann

LZN Laser Zentrum Nord GmbH

Tobias Fritze

EDAG Engineering $\mathrm{GmbH}$

Kai Geißenhöner

Dr. Ing. h.c. F. Porsche AG

Prof. Dr. Lothar Harzheim

Adam Opel AG

Heiko Herchet

trive.me - eine Marke

der EDAG Engineering $\mathrm{GmbH}$

Dr. Martin Hillebrecht

EDAG Engineering $\mathrm{GmbH}$

Helge Kiebach

KTI GmbH \& Co. KG

Dr. Roland Krivachy

MAN Truck \& Bus AG

Oliver Mende

Volkswagen AG
Andreas Orf

AUDI AG

Detlev Ranft

Dr. Ing. h.c. F. Porsche AG

Prof. Dr. Klemens Rother

Fakultät Maschinenbau /

Fahrzeugtechnik / Flugzeugtechnik, Hochschule München

\section{Robert N. Saje}

General Motors Company, USA

Frank Saterdag

Adam Opel AG

Ulrich Sieg

ehem. Hamburger Hochbahn AG

\section{Sylvain Wehnert}

Daimler AG

\section{Sascha Wörner}

Kompetenzzentrum

für Verkehr - Mobilität - Automotive,

Technische Hochschule Mittelhessen

Dr. Wolfgang Wohlers

Airbus Operations $\mathrm{GmbH}$

Dr. Lars Woyna

Adam Opel AG

Klaus Zander

EDAG Engineering $\mathrm{GmbH}$

Rolf Züchner

Ford-Werke $\mathrm{GmbH}$ 